\title{
Herramientas Digitales para Entornos Educativos Virtuales
}

\section{Digital Tools for Virtual Educational Environments}

Flor de María Ccoa Mamani* https://orcid.org/0000-0001-8881-9640 Cleofé Genoveva Alvites-Huamaní** https://orcid.org/0000-0001-6328-6470 http://dx.doi.org/10.21503/lex.v19i27.2265

* Estudiante del Doctorado en educación de la Universidad César Vallejo-SP, Magíster en administración de la educación, Licenciada en Educación Inicial, especialista en Educación en UGEL Canas-Cusco-Perú. Estudiante adscrita al proyecto PIPD, Innovación docente y uso de las Tecnologías de la Información y Comunicación en el proceso de enseñanza-aprendizaje. Perú.

Correo electrónico: fccoa@ucvvirtual.edu.pe

** Docente de la Escuela de Posgrado de la Universidad César Vallejo, Editor en Jefe de la Revista Hamut ay-Universidad Alas Peruanas. Perú.

Correo electrónico: acleofe@ucv.edu.pe

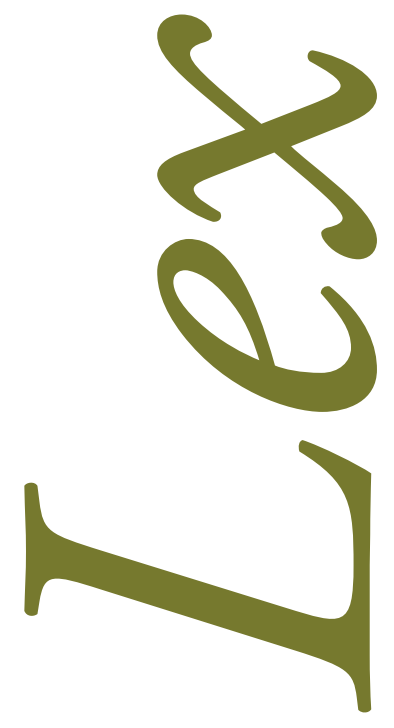




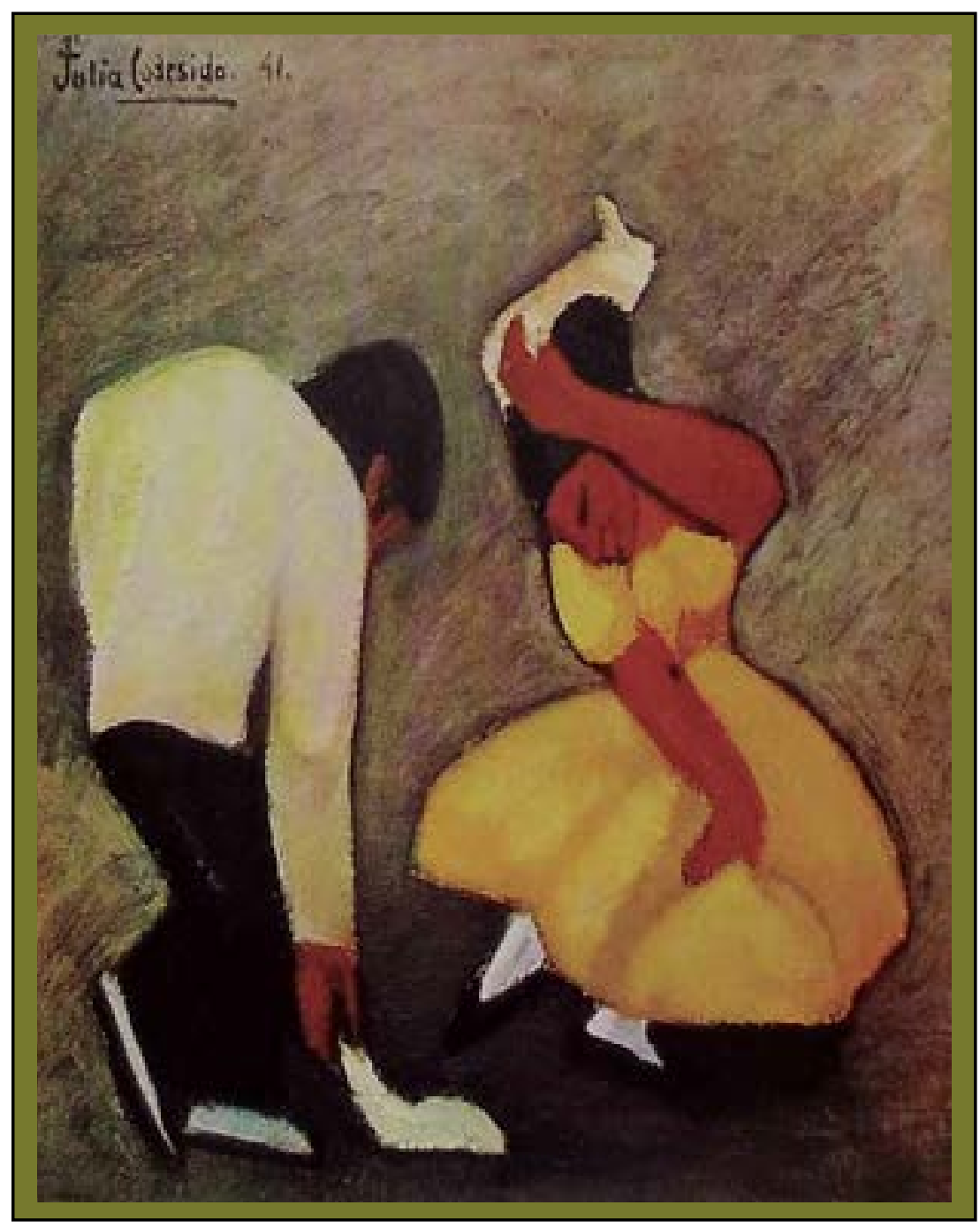

Marinera. Colección particular. Julia Codesido pintora peruana del indigenismo. (Lima 1883-1979). 


\section{RESUMEN}

La relevancia alcanzada por las tecnologías a nivel mundial a causa de la pandemia ha conllevado a un constante incremento en el uso de las herramientas digitales en los entornos educativos virtuales. Esto debido a que la educación ha dejado de ser presencial y pasó a convertirse en educación remota en su totalidad. En razón de ello, este estudio tuvo como objetivo revisar la literatura sobre las herramientas digitales para analizar su incidencia en los entornos digitales. La metodología utilizada fue descriptiva documental y se utilizaron diversas bases de datos que permitieron recopilar la información. De un total de 81 artículos seleccionados, se depuraron 59, quedando 22. Los resultados encontrados enfatizan en que los docentes deben aplicar las TIC en sus sesiones de aprendizaje, dado que las herramientas digitales son importantes e imprescindibles dentro del proceso de enseñanza-aprendizaje. Igualmente, se advirtió que se ha incrementado el uso de herramientas digitales en sus diversas funcionabilidades.

Palabras clave: Tecnologias, herramientas digitales, educación online, virtualidad.

\section{ABSTRACT}

The relevance achieved by technologies worldwide due to the pandemic has led to a constant increase in the use of digital tools in virtual educational environments. This is due to the fact that education has ceased to be face-to-face and has become entirely remote education. Therefore, the goal of this study was to review the literature on digital tools to analyze their incidence in digital environments. The method used was descriptive documentary and various databases were used to collect the information. Of a total of 81 articles selected, 59 were filtered, leaving 22 . The results found emphasize that teachers should apply ICT in their learning sessions, since digital tools are important and essential in the teaching-learning process. Likewise, it was noted that the use of digital tools has increased in its various functions.

Key words: Technologies, digital tools, online education, virtuality. 


\section{INTRODUCCIÓN}

El importante avance científico tecnológico a nivel mundial ha traído trascendentales cambios en el modo de vivir del ser humano y de la sociedad en general, (Gómez et al. ${ }^{1}$, Gargallo ${ }^{2}$, Rizales-Semprum et al. ${ }^{3}$, Didier et al. $\left.{ }^{4}\right)$. Esto ha conllevado a la masificación del conocimiento y de las telecomunicaciones y permitido que millones de personas accedan a herramientas tecnológicas, sea físicas o virtuales. Así se han facilitado la realización eficiente de tareas que anteriormente demandaban mucha inversión de tiempo y esfuerzo y que, en muchos casos, no se podían solucionar. Pero en los complicados tiempos de la emergencia sanitaria, el auge de las tecnologías de la información y comunicación (TIC) cobró importancia en todos los campos (académicos, culturales, sociales y empresariales) y muchos países se vieron obligados a masificar el uso de las mismas, considerando la imposibilidad del contacto físico. Esto también se ha visto reflejado en la educación, que experimentó un drástico cambio, al pasar de una educación tradicional donde no estaba incluida las TIC a una educación totalmente virtualizada. En estas circunstancia toda la comunidad educativa no tuvo más opción que adaptarse a esta nueva manera de enseñanza, en las que las TIC cumplen un papel preponderante, pues ahora son fundamentales en el proceso de aprendizaje para la vida, (Melo et al. ${ }^{5}$ ).

Esta situación se debe principalmente a que las TIC se posicionaron como una herramienta imprescindible para la enseńanza virtual; facilitan la realización de tareas, promueven la inclusión y fomentan

1. Carlos Alberto Gómez, Verenice Sánchez and Libardo Ramón. "Incorporar las TIC a los procesos de enseñanza-aprendizaje". Horizontes Pedagógicos 19.1 (2017): 48-54.

2. Ana Felicitas Gargallo "La integración de las TIC en los procesos educativos y organizativos". Educar em Revista 69 (2018): 325-339.

3. Mervin Rizales-Semprum, Carlos Gómez-Valderrama, and César Hernández-Suarez. "Uso de herramientas tecnológicas para la enseñanza de las ciencias en educación media diversificada de acuerdo a la modalidad de estudio a distancia”. Eco matemático 10.2 (2019): 35-46.

4. Lucila Didier, Verónica Ficoseco, and Edvaldo Souza. "El Programa Avanzado de Educación Secundaria con énfasis en Tecnologías de Información y Comunicación (PROA) y su presencia en Facebook". Revista Latinoamericana de Tecnología Educativa 19.1 (2020): 107-120.

5. Eugenia Melo, Juan Llopis, José Gascó, and Reyes González. "Integration of ICT into the higher education process: The case of Colombia”. Journal of Small Business Strategy 30.1 (2020): 58-67. 
la comunicación entre los discentes y entre estos y los docentes; además, permiten una comunicación en diversos ámbitos geográficos, (Alderete y Formichella ${ }^{6}$.

La incursión de las TIC ha incidido en el perfil del docente, (Gómez et al. ${ }^{7}$, Berrios-Aguayo et al. ${ }^{8}$, Monsalve-Lorente y Aguasanta-Regalado ${ }^{9}$ ). estos tuvieron que reinventar su trabajo pedagógico, lo que implicó que apliquen una serie de estrategias para la integración de las TIC con los modelos educativos vigentes. Y es absolutamente necesario, (Monteiro et al. ${ }^{10}$, Cosi et al. ${ }^{11}$, Orellana-Fernández et al. ${ }^{12}$,) porque todos los nińos y jóvenes en etapa escolar son nativos digitales y viven rodeados permanentemente por elementos de carácter tecnológico (en los planos físico o virtual). Esta situación se constata en el hecho de que ellos usan la tecnología sin temor y de forma natural. En ese contexto, el modo en que aprenden, y cómo se les puede enseñar, debe involucrar necesariamente un escenario que implique el uso de herramientas tecnológicas, (Cosi et al. ${ }^{13}$ ), considerando que la educación en muchos casos esta enmarcada dentro del enfoque educativo constructivista, puesto que promueven la construcción del conocimiento en forma colaborativa. De esta manera los estudiantes logran aprendizajes más significativos en estos entornos educativos generados por TIC, los cuales también se encuentran enmarcados dentro de este mismo enfoque, (Aparicio ${ }^{14}$ ). Las TIC son un cúmulo de herramientas digitales diseñadas para gestionar, almacenar, generar, difundir e interactuar una serie de información en el ciberespacio que están vinculadas para el uso en entornos educativos virtuales, (Orellana-Fernández et al. ${ }^{15}$ ), las cuales inciden en el entorno educativo, $\left(\right.$ Monzón $\left.{ }^{16}\right)$.

6. María Verónica Alderete, and María Marta Formichella. "El acceso a las TIC en el hogar y en la escuela: su impacto sobre los logros educativos”. Revista de Economía del Rosario 19.2 (2016): 221-242.

7. Ibidem, 1

8. Beatriz Berrios-Aguayo, María Molina-Jaén, and Antonio Pantoja-Vallejo, "Opinion of ICT coordinators on the incidence of telematic tools". Journal of Technology and Science Education 10.1 (2020): 142-158.

9. Laura Monsalve-Lorente, and Miriam Aguasanta-Regalado. "Nuevas ecologías del aprendizaje en el currículo: la era digital en la escuela”. Revista Latinoamericana de Tecnología Educativa 19.1 (2020): 139-154.

10. Larisse Monteiro, Bruno Rodrigues da Costa, Valdinei Gomes, and Talita Carvalho. "O uso do software operações com números naturais como ferramenta facilitadora para o processo de ensino das quatro operações: adição, subtração, multiplicação e divisão". Paradigma 41.2 (2020): 550-572.

11. Alexandra Cosi, Núria Voltas, José Lázaro-Cantabrana, Paula Morales, Mireia Calvo, Silvia Molina, and María Ángeles Quiroga. "Formative assessment at university using digital technology tools". Revista de currículum y formación del profesorado 24.1 (2020): 164-183.

12. Oscar Orellana-Fernández, Darwin García-Herrera, Ana Castro-Salazar, and Juan Erazo-Álvarez. "Iniciación a la lectura a través de las TIC: Una propuesta metodológica”. Revista Arbitrada Interdisciplinaria KOINONIA 5.1 (2020): 673-691.

13 Ibidem, 11

14. Oscar Yecid Aparicio. "Las TIC como herramientas cognitivas". Revista interamericana de investigación, educación y pedagogía 11.1 (2018): 67-80.

15. Ibidem, 12

16. Elba Marina Monzón. "Alfabetización digital en el aula". En Proceedings of the Digital World Learning Conference-CIEV 2019 (2020): 89-98. 
En base a lo expuesto, este artículo tuvo como objetivo aportar información sobre las herramientas digitales para entornos virtuales educativos, a través de una revisión de la literatura.

\section{MÉTODO}

El estudio es de tipo descriptivo-documental. Se realizó mediante la revisión de la literatura entre los años 2015 y 2021. Las bases de datos utilizadas fueron: Ebsco (36\%), Scopus (18\%), Dialnet y Redalyc (14\%), Scielo y Google Académico (9\%), (Figura 2); el 75\% fueron en español, el 20\% en inglés y el $5 \%$ en portugués (Figura 3). Para una búsqueda específica, se utilizaron palabras claves como: Herramientas TIC, recursos, Technology, entornos virtuales, educación y TIC. La Figura 1 describe el proceso realizado para la revisión de la literatura, (Hilario, Izquierdo y Ríos ${ }^{17}$ ).

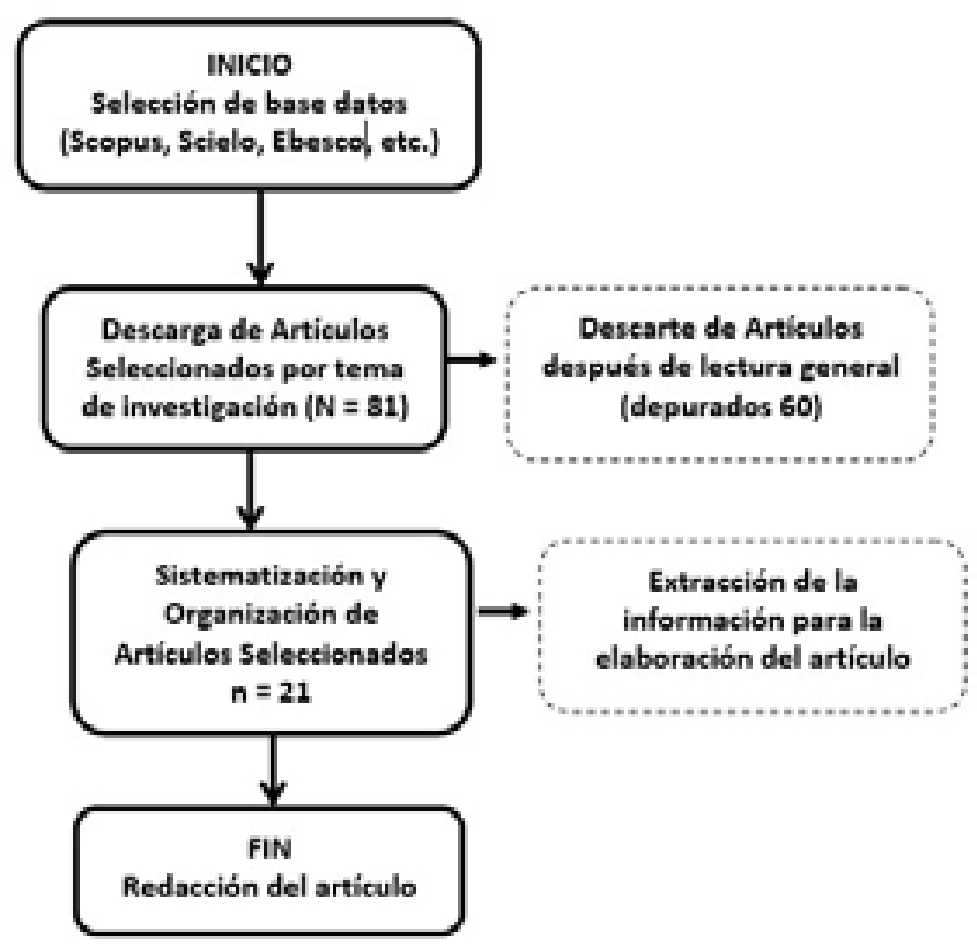

Figura 1. Proceso para la revisión de la literatura

Fuente: Elaboración propia (2021)

17. Gina Hilario, Joana Izquierdo, and Claudia Ríos. "Dependencia emocional y su relación con la violencia en parejas. Una aproximación descriptiva a la revisión de literatura”. Revista Desafíos 11.2 (2020): 120-5. 


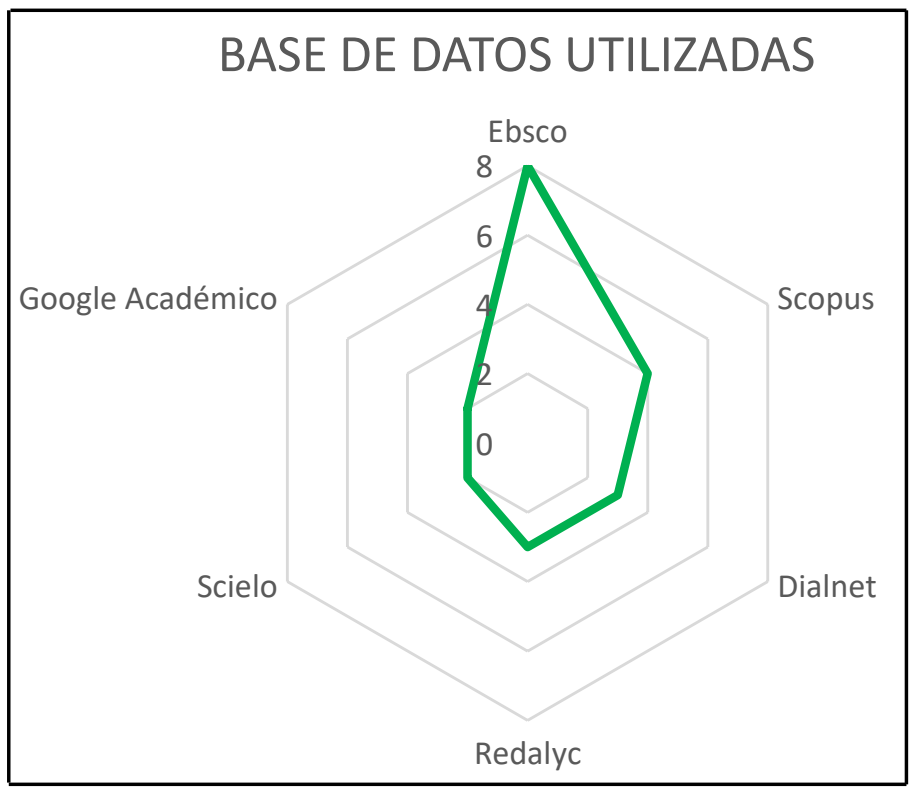

Figura 2. Bases de datos utilizadas para recopilar información

Fuente: Elaboración propia (2021)

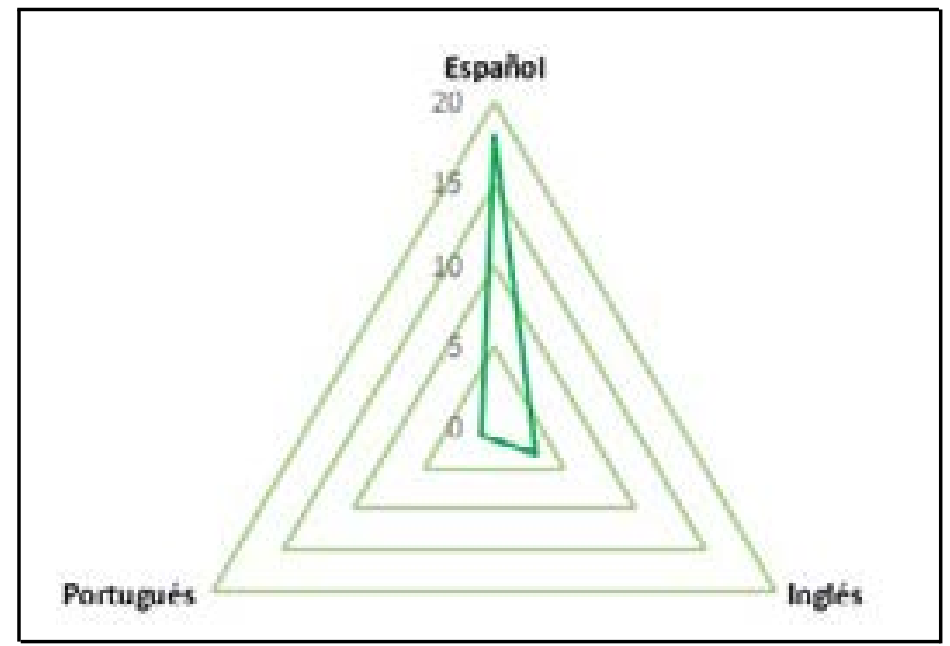

Figura 3.Idioma de los documentos analizados

Fuente: Elaboración propia (2021) 


\section{HERRAMIENTAS DIGITALES}

En los últimos años, las TIC jugaron un papel preponderante en todos los aspectos que involucran al ser humano, en lo económico, social, y educativo (Lorduy y Naranjo ${ }^{18}$ ). En ese entender, las TIC motivaron una transformación transcendental en el rol que desempeñan tanto el docente como los estudiantes en el proceso de aprendizaje-enseñanza, en todas las etapas, modalidades, y niveles educativos (Báez y Clunie ${ }^{19}$ Rizales-Semprum et al. ${ }^{20}$ ). En ese sentido, la utilización de herramientas tecnológicas digitales, por parte de los docentes, durante las sesiones de aprendizaje, generó en los estudiantes una especial motivación y mayor interés en la gestión de su propio aprendizaje. Esto conllevó al logro de mejores resultados escolares (Monteiro et al. ${ }^{21}$ Vargas-Murillo ${ }^{22}$, Orellana-Fernández et al. ${ }^{23}$ ). La utilización de herramientas tecnológicas en la enseñanza-aprendizaje, facilita la gestión de la información (recopilación, análisis, almacenamiento, producción y difusión), (Rizales-Semprum et al., ${ }^{24}$ ).

El uso de herramientas TIC en el proceso de aprendizaje-enseñanza favorece el desarrollo de una buena comunicación entre el docente y los estudiantes, como también entre estos últimos (Rizales-Semprum et al..$^{25}$ Orellana-Fernández et al. ${ }^{26}$ Lorduy y Naranjo ${ }^{27}$ ). El trabajo pedagógico aprovechando recursos y herramientas tecnológicas fomenta el desarrollo de un pensamiento crítico y promueve el logro de aprendizajes en forma autónoma y colaborativa, (Formichella y Alderete ${ }^{28}$ ). El uso de las TIC puede tener un efecto positivo en el futuro económico, productivo, laboral, y social de los estudiantes, siempre y cuando se aprovechen en actividades educativas que les sea funcionales. De la misma manera, García et al. ${ }^{29}$ Arancibia et al. ${ }^{30}$, refieren que el uso de la tecnología en el proceso de enseñanza-aprendizaje juega un papel determinante en la construcción del conocimiento y el logro de competencias de los estudiantes.

18. Danny Lorduy, and Claudia Naranjo. “Tecnologías de la información y la comunicación aplicadas a la educación en ciencias. Praxis \& Saber 11.27 (2020): 58-67.

19. Carmen Inés Báez, and Clifton Clunie. "Una mirada a la Educación Ubicua”. RIED. Revista Iberoamericana de Educación a Distancia 22.1 (2019): 325-344.

20. Ibidem, 3

21. Ibidem, 10

22. Gavino Vargas-Murillo. "Estrategias educativas y tecnología digital en el proceso enseñanza aprendizaje”. Cuadernos 61.1 (2020): 69-76.

23. Ibidem, 12

24. Ibidem, 3

25. Ibidem, 3

26. Ibidem, 12

27. Ibidem, 18

28. María Marta Formichella, and María Verónica Alderete. “TIC en la escuela y rendimiento educativo: el efecto mediador del uso de las TIC en el hogar". Cuadernos de Investigación Educativa 9.1 (2018): 75-93.

29. Lorenzo García Aretio. “COVID-19 y educación a distancia digital: preconfinamiento, confinamiento y posconfinamiento. Revista Iberoamericana de Educación a Distancia 24.1 (2021): 9-25.

30. María Arancibia, Julio Cabero, and Verónica Marín. “Creencias sobre la enseñanza y uso de las tecnologías de la información y la comunicación (TIC) en docentes de educación superior”. Formación Universitaria 13.3 (2020): 89-100. 
Para Quintana y $\mathrm{Bo}^{31}$ el trabajo pedagógico con mediación de las TIC puede presentarse de cuatro formas, (Figura 4).

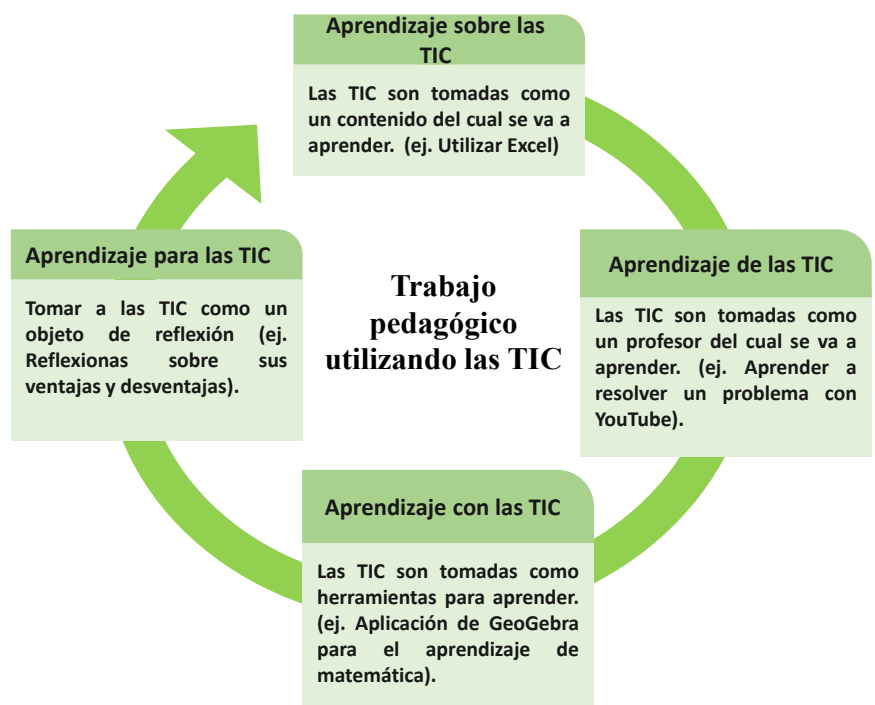

Figura 4. Trabajo pedagógico utilizando las TIC.

Fuente: Quintana y Bo, (2015)

Aparicio $^{32}$ menciona que para aprender con la tecnología implica que en el proceso de enseñanza-aprendizaje se debe implementar herramientas tecnológicas que vinculen lo cognitivo. Para esto, el docente contará con las competencias suficientes para acompañar al estudiante en la construcción de su aprendizaje. También, incorporar las TIC a la evaluación se traducirá en el logro de mejores resultados de aprendizaje, (Formichella y Alderete ${ }^{33}$ ), puesto que el estudiante es el propio protagonista de su proceso evaluativo. $\mathrm{Al}$ respecto Cosi et al. ${ }^{34}$, afirman que la utilización de herramientas tecnológicas durante el proceso de aprendizaje-enseñanza propicia una evaluación formativa, por diversas razones, como se puede observar en la Tabla 1.

31. Jordi Quintana, and Imma Bo. "La escuela inclusiva: Concepciones, prácticas, profesorado y tecnologías". Nuances: estudos sobre Educação, Presidente Prudente-SP 26.2 (2015): 93-111.

32. Ibidem, 14

33. Ibidem, 28

34. Ibidem, 11 
Tabla 1. Razones para Utilizar Herramientas TIC en la Evaluación

\begin{tabular}{ll}
\hline \multicolumn{1}{c}{ Aspectos } & \multicolumn{1}{c}{ Razones } \\
\hline Etapas & $\begin{array}{l}\text { La evaluación utilizando recursos tecnológicos puede darse } \\
\text { durante todo el proceso de enseñanza, es decir: al inicio, durante } \\
\text { el proceso y al cierre del proceso. }\end{array}$ \\
\hline Tipos & $\begin{array}{l}\text { La evaluación utilizando recursos tecnológicos puede darse de } \\
\text { diversos tipos como pueden ser: heteroevaluación, coevaluación } \\
\text { y, sobre todo, autoevaluación. }\end{array}$ \\
\hline Tiempo & $\begin{array}{l}\text { Los recursos tecnológicos permiten que la evaluación y su } \\
\text { respectiva retroalimentación deben darse de forma inmediata. }\end{array}$ \\
\hline Amplitud & $\begin{array}{l}\text { Los recursos tecnológicos son tan amplios y diversos que } \\
\text { permiten que la evaluación se realice mediante textos, audios, } \\
\text { videos, juegos y otros. }\end{array}$ \\
\hline Objetividad & $\begin{array}{l}\text { Por sus características, una evaluación realizada con recursos } \\
\text { tecnológicos es netamente objetiva. }\end{array}$ \\
\hline
\end{tabular}

Fuente: Adaptado de Cosi et al. (2020).

\section{HERRAMIENTAS DIGITALES PARA ENTORNOS VIRTUALES EN EDUCACIÓN}

Existen una infinidad de herramientas tecnológicas digitales diseñadas exclusivamente para que sirvan como recursos para el proceso de enseñanza-aprendizaje de los estudiantes. También hay un sinnúmero de herramientas TIC que originalmente no fueron diseñadas con fines pedagógicos; sin embargo, con una previa y adecuada planificación, fueron acondicionadas y utilizadas para lograr aprendizajes de calidad en los estudiantes.

Vargas-Murillo ${ }^{35}$ hace referencia a algunas herramientas TIC que se pueden aprovechar en el trabajo pedagógico, (Tabla 2).

35. Ibidem, 22 


\section{Tabla 2. Herramientas Tecnológicas Digitales Aplicables a entornos virtuales en educación}

\begin{tabular}{|c|c|c|c|}
\hline $\begin{array}{c}\text { Tipo de Herramienta } \\
\text { Tecnológica }\end{array}$ & $\begin{array}{c}\text { Nombre de } \\
\text { Herramienta } \\
\text { Tecnológica } \\
\end{array}$ & $\begin{array}{l}\text { Aprovechamiento } \\
\text { Pedagógico. }\end{array}$ & Enlace \\
\hline $\begin{array}{l}\text { Software para gestión de } \\
\text { mapas mentales }\end{array}$ & XMind & $\begin{array}{l}\text { Se utiliza para elaborar mapas } \\
\text { mentales, mapas } \\
\text { conceptuales. }\end{array}$ & https://www.xmind.net/download/ \\
\hline $\begin{array}{l}\text { Software para gestión de } \\
\text { mapas conceptuales }\end{array}$ & Cmaps Tools & $\begin{array}{l}\text { Se utiliza para elaborar mapas } \\
\text { conceptuales. }\end{array}$ & https://cmap.ihmc.us/cmaptools/ \\
\hline $\begin{array}{l}\text { Software para gestión de } \\
\text { actividades educativas }\end{array}$ & Hot Potatoes & $\begin{array}{l}\text { Se utiliza para realizar } \\
\text { actividades educativas } \\
\text { interactivas sencillas }\end{array}$ & https://hotpot.uvic.ca/ \\
\hline $\begin{array}{l}\text { Software para gestión de } \\
\text { actividades educativas }\end{array}$ & JClic & $\begin{array}{l}\text { Se utiliza para realizar } \\
\text { actividades educativas } \\
\text { interactivas }\end{array}$ & https://clic.xtec.cat/legacy/es/jclic/ \\
\hline $\begin{array}{l}\text { Plataforma Virtual } \\
\text { Educativa }\end{array}$ & Google Classroom & $\begin{array}{l}\text { Plataforma exclusiva para } \\
\text { gestionar recursos educativos. }\end{array}$ & https://classroom.google.com/ \\
\hline $\begin{array}{l}\text { Plataforma Virtual } \\
\text { Educativa }\end{array}$ & Schoology & $\begin{array}{l}\text { Plataforma para gestionar } \\
\text { recursos educativos. }\end{array}$ & https://www.schoology.com/ \\
\hline $\begin{array}{l}\text { Plataforma Virtual } \\
\text { Educativa }\end{array}$ & Moodle & $\begin{array}{l}\text { Plataforma que crea } \\
\text { completos ambientes } \\
\text { educativos. }\end{array}$ & https://moodle.org/ \\
\hline $\begin{array}{l}\text { Plataforma Virtual para la } \\
\text { gestión de encuestas }\end{array}$ & Google Forms & $\begin{array}{l}\text { Se puede utilizar para realizar } \\
\text { evaluaciones, así como } \\
\text { también para aplicar } \\
\text { encuestas }\end{array}$ & $\begin{array}{l}\text { https://www.google.com/intl/es- } \\
419 \text { pe/forms/about/ }\end{array}$ \\
\hline $\begin{array}{l}\text { Plataforma para compartir } \\
\text { todo tipo de contenidos }\end{array}$ & Facebook & $\begin{array}{l}\text { Se puede utilizar } \\
\text { pedagógicamente por medio } \\
\text { de grupos cerrados exclusivos } \\
\text { para el aprendizaje. Gestiona } \\
\text { de manera eficiente la } \\
\text { información }\end{array}$ & https://www.facebook.com \\
\hline $\begin{array}{l}\text { Aplicación móvil de } \\
\text { mensajería instantánea }\end{array}$ & WhatsApp & $\begin{array}{l}\text { Se puede utilizar } \\
\text { pedagógicamente por medio } \\
\text { de grupos exclusivos para el } \\
\text { aprendizaje. Gestiona de } \\
\text { manera eficiente la } \\
\text { retroalimentación }\end{array}$ & https://web.whatsapp.com/ \\
\hline $\begin{array}{l}\text { Plataforma para } \\
\text { videoconferencias }\end{array}$ & Zoom & $\begin{array}{l}\text { Se puede utilizar para realiza } \\
\text { sesiones de aprendizaje en } \\
\text { vivo mediante } \\
\text { videoconferencia. }\end{array}$ & https://zoom.us/ \\
\hline $\begin{array}{l}\text { Plataforma para reuniones } \\
\text { en línea }\end{array}$ & Google Meet & $\begin{array}{l}\text { Se puede utilizar para realiza } \\
\text { sesiones de aprendizaje en } \\
\text { vivo mediante } \\
\text { videoconferencia. }\end{array}$ & https://meet.google.com/ \\
\hline $\begin{array}{l}\text { Plataforma para gestionar } \\
\text { infografías }\end{array}$ & Easelly & $\begin{array}{l}\text { Se puede diseñar atractivas } \\
\text { infografias con respecto a un } \\
\text { tema específico }\end{array}$ & https://www.easel.ly/ \\
\hline $\begin{array}{l}\text { Plataforma para gestionar } \\
\text { infografías }\end{array}$ & Picktochart & $\begin{array}{l}\text { Se puede diseñar atractivas } \\
\text { infografías con respecto a un } \\
\text { tema específico }\end{array}$ & https://create.piktochart.com/ \\
\hline Software de oficina & Office Word & $\begin{array}{l}\text { Sirve para gestión de } \\
\text { documentos en actividades de } \\
\text { producción de textos }\end{array}$ & https://office.live.com/ \\
\hline
\end{tabular}




\begin{tabular}{lcll}
\hline Software para evaluación & Socrative & $\begin{array}{l}\text { Sirve para gestionar } \\
\text { evaluaciones }\end{array}$ & www.socrative.com \\
\hline $\begin{array}{l}\text { Herramienta para } \\
\text { retroalimentación }\end{array}$ & Kahoot & $\begin{array}{l}\text { El recurso facilita el } \\
\text { reforzamiento }\end{array}$ & www.kahoot.com \\
\hline $\begin{array}{l}\text { Recurso para compartir } \\
\text { todo tipo de contenidos }\end{array}$ & Padlet & $\begin{array}{l}\text { Se utiliza para realizar } \\
\text { actividades educativas } \\
\text { interactivas sencillas }\end{array}$ & www.padlet.com \\
\hline $\begin{array}{l}\text { Plataforma Virtual para la } \\
\text { gestión de encuestas }\end{array}$ & Mentimenter & $\begin{array}{l}\text { Se puede utilizar para realizar } \\
\text { evaluaciones, así como } \\
\text { también para aplicar } \\
\text { encuestas }\end{array}$ & https://www.mentimeter.com/ \\
\hline
\end{tabular}

Fuente: Elaboración propia (2021), adaptado de Vargas-Murillo.

García $^{36}$ refiere que las herramientas digitales publicadas en la web facilitaron la docencia en línea; además, el uso de estas facilita y viabiliza la enseñanza por la edad de los estudiantes, quienes desde años atrás tienen la posibilidad de interacción con las TIC. Las herramientas digitales brindan una gama de posibilidades a los entornos educativos virtuales, si estás son bien direccionadas por el docente, (Monsalve-Lorente y Aguasanta-Regalado ${ }^{37}$ ). Monteiro et al. ${ }^{38}$, Cosi et al. ${ }^{39}$, Orellana-Fernández et al. ${ }^{40}$, coinciden en mencionar que en la actualidad los niños y jóvenes en etapa escolar son nativos digitales, interactúan continuamente con dispositivos electrónicos y aplicaciones digitales. Al respecto, Garga$1 \mathrm{lo}^{41}$ señala que, de un tiempo a esta parte, se produjo un incremento notable del uso de la tecnología en el campo educativo. Para García ${ }^{42}$ la pandemia obligó al desarrollo de mayor cantidad de plataformas, recursos y herramientas digitales para el proceso de enseñanza-aprendizaje, (Figura 5).

36. Ibidem, 29

37. Ibidem, 9

38. Ibidem, 10

39. Ibidem, 11

40. Ibidem, 12

41. Ibidem, 2

42. Ibidem, 29 


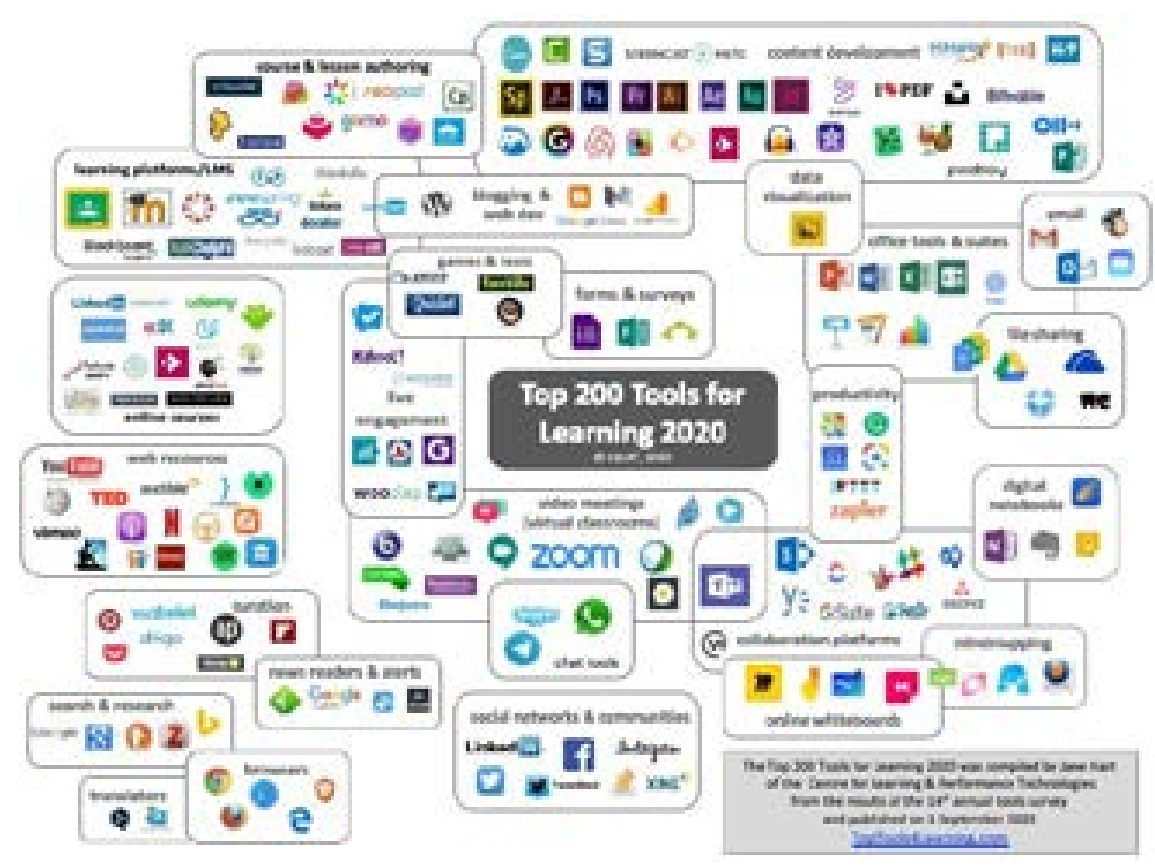

Figura 5. Principales herramientas Web utilizadas en 2020.

Fuente: García (2021)

\section{DISCUSIÓN Y CONCLUSIONES}

Las Tecnologías de la Información y Comunicación son un grupo de recursos tecnológicos digitales cuyo fin primordial es gestionar la información, permitiendo el crear, recoger, almacenar y difundir la información. La apropiación de las TIC de una manera abrupta en la educación provocó un cambio fundamental en la enseñanza a nivel mundial, debido a la pandemia. En la medida de que todos los niños y jóvenes en edad escolar son nativos digitales, la utilización de las TIC como herramienta en los procesos de aprendizaje-enseñanza posibilitó que los educandos se motiven más para gestionar su propio aprendizaje. Para Monteiro et al. ${ }^{43}$, Vargas-Murillo ${ }^{44}$ Orellana-Fernández et al. ${ }^{45}$, estas propiciaron mejores resultados de aprendizaje escolar. Visto así, el uso de herramientas TIC en el proceso de aprendizaje-enseñanza potencia en los discentes el desarrollo de competencias relacionadas con la comunicación y el pensamiento crítico; ellos mismos construyen su aprendizaje de manera autónoma y colaborativa, según García et al. ${ }^{46}$, y Arancibia et al. ${ }^{47}$

43. Ibidem, 10

44. Ibidem, 22

45. Ibidem, 12

46. Ibidem, 29

47. Ibidem, 30 
En base a las múltiples investigaciones que existe sobre el particular, se puede concluir que el uso de herramientas tecnológicas en la escuela beneficia el aprendizaje-enseñanza. Los estudiantes se motivan a seguir aprendiendo. Por añadidura, se propicia la evaluación formativa y el desarrollo autónomo de competencias. En suma, esto se traduce en el logro de mejores resultados de aprendizaje. La variedad de herramientas TIC digitales (llámese software) puede utilizarse como recursos en el proceso de aprendizaje-enseñanza. Se puede mencionar, algunas de estas herramientas XMind, Cmaps Tools, Hot potatoes, JClic, Google Classroom, Schoology, Moodle, Google Forms, Facebook, WhatsApp, Zoom, Google Meet, Easelly, Picktochart, Office Word, Office PowerPoint, que pueden apoyar el activar procesos de aprendizaje exitosos con mediación de las TIC, pero la decisión y selección corresponde a los docentes. Por su parte, los estudiantes tienen que desarrollar la habilidad para aprovecharlas en la realización creativa y productiva de las actividades de aprendizaje propuestas por aquellos.

\section{AGRADECIMIENTO}

El estudio forma parte del proyecto PIPD, Innovación docente y uso de las Tecnologías de la Información y Comunicación en el proceso de enseñanza aprendizaje, aprobado con Resolución de Dirección Académica No. 012 - 2020-DA-UCV de la Escuela de Posgrado de la Universidad César Vallejo-SP.

\section{REFERENCIAS}

- $\quad$ Alderete, María Verónica and Formichella, María Marta. "El acceso a las TIC en el hogar y en la escuela: su impacto sobre los logros educativos". Revista de Economía del Rosario 19.2 (2016): 221-242. http://dx.doi.org/10.12804/revistas.urosario.edu.co/economia/a.5626

- Arancibia, María, Cabero, Julio, and Marín, Verónica. "Creencias sobre la enseñanza y uso de las tecnologías de la información y la comunicación (TIC) en docentes de educación superior”. Formación Universitaria 13.3 (2020): 89-100. http://dx.doi.org/10.4067/S0718-50062020000300089

- Aparicio, Oscar Yecid. "Las TIC como herramientas cognitivas". Revista interamericana de investigación, educación y pedagogía 11.1 (2018): 67-80. http://www.redalyc.org/articulo.oa?id=561059324005

- Báez, Carmen Inés and Clunie, Clifton. "Una mirada a la Educación Ubicua”. RIED. Revista Iberoamericana de Educación a Distancia 22.1 (2019): 325-344. http://dx.doi.org/10.5944/ried.22.1.22422

- Berrios-Aguayo, Beatriz, Molina-Jaén, María and Pantoja-Vallejo, Antonio. "Opinion of ICT coordinators on the incidence of telematic tools". Journal of Technology and Science Education 10.1 (2020): 142-158. https://doi.org/10.3926/jotse.690 
- Cosi, Alexandra., Voltas, Núria, Lázaro-Cantabrana, José, Morales, Paula, Calvo, Mireia, Molina, Silvia, and Quiroga, María Ángeles. "Formative assessment at university using digital technology tools”. Revista de currículum y formación del profesorado 24.1 (2020): 164-183. https://doi.org/10.30827/profesorado.v24i1.9314

- $\quad$ Didier, Lucila, Ficoseco, Verónica. and Souza, Edvaldo. "El Programa Avanzado de Educación Secundaria con énfasis en Tecnologías de Información y Comunicación (PROA) y su presencia en Facebook". Revista Latinoamericana de Tecnología Educativa 19.1 (2020): 107-120. http://dx.doi.org/10.17398/1695-288X.19.1.107

- Formichella, María Marta. and Alderete, María Verónica. "TIC en la escuela y rendimiento educativo: el efecto mediador del uso de las TIC en el hogar". Cuadernos de Investigación Educativa 9.1 (2018): 75-93. https://doi.org/10.18861/cied.2018.9.1.2822

- García Aretio, Lorenzo. "COVID-19 y educación a distancia digital: preconfinamiento, confinamiento y posconfinamiento. Revista Iberoamericana de Educación a Distancia 24.1 (2021): 9-25. https://doi.org/10.5944/ried.24.1.28080

- García, Maritza, Deco, Claudia, Bender, Cristina and Collazos, César. "Herramientas de Diseño para el Desarrollo de Competencias en Educación Básica, Media y Tecnológica: Experiencia en el Instituto Técnico Industrial Pascual Bravo de Colombia”. Revista Iberoamericana de Educación en Tecnología y Tecnología en Educación 19 (2017): 73-82. https://doi.org/10.24215/18509959.0.p.\%2073-82

- Gargallo, Ana Felicitas. "La integración de las TIC en los procesos educativos y organizativos". Educar em Revista 69 (2018): 325-339. http://www.redalyc.org/articulo.oa?id=155059187017

- Gómez, Carlos Alberto, Sánchez, Verenice, and Ramón, Libardo. "Incorporar las TIC a los procesos de enseñanza-aprendizaje”. Horizontes Pedagógicos 19.1 (2017): 48-54. https://revistas.iberoamericana.edu.co/index.php/rhpedagogicos/article/view/1138

- Hilario, Gina, Izquierdo, Joana and Ríos, Claudia. "Dependencia emocional y su relación con la violencia en parejas. Una aproximación descriptiva a la revisión de literatura". Revista Desafios 11.2 (2020): 120-5. https://doi.org/10.37711/desafios.2020.11.2.211

- Lorduy, Danny and Naranjo, Claudia. "Tecnologías de la información y la comunicación aplicadas a la educación en ciencias. Praxis \& Saber 11.27 (2020): 58-67. https://doi.org/10.19053/22160159.v11.n27.2020.11177 
- Melo, Eugenia., Llopis, Juan, Gascó, José. and González, Reyes. "Integration of ICT into the higher education process: The case of Colombia”. Journal of Small Business Strategy 30.1 (2020): 58-67. https://libjournals.mtsu.edu/index.php/jsbs/article/view/1665

- $\quad$ Monteiro, Larisse, Rodrigues da Costa, Bruno, Gomes, Valdinei, and Carvalho, Talita. "O uso do software operaçóes com números naturais como ferramenta facilitadora para o processo de ensino das quatro operaçôes: adição, subtração, multiplicação e divisão". Paradigma 41.2 (2020): 550-572. http://revistaparadigma.online/ojs/index.php/paradigma/article/view/918

- Monsalve-Lorente, Laura. and Aguasanta-Regalado, Miriam. "Nuevas ecologías del aprendizaje en el currículo: la era digital en la escuela". Revista Latinoamericana de Tecnologia Educativa 19.1 (2020): 139-154. http://dx.doi.org/10.17398/1695-288X.19.1.139

- Monzón, Elba Marina. "Alfabetización digital en el aula”. En Proceedings of the Digital World Learning Conference-CIEV 2019 (2020): 89-98.

http://biblioteca.galileo.edu/tesario/handle/123456789/960

- Orellana-Fernández, Oscar, García-Herrera, Darwin, Castro-Salazar, Ana and Erazo-Álvarez, Juan. "Iniciación a la lectura a través de las TIC: Una propuesta metodológica".

Revista Arbitrada Interdisciplinaria KOINONIA 5.1 (2020): 673-691. http://dx.doi.org/10.35381/r.k.v5i1.804

- Quintana, Jordi. and Bo, Imma. "La escuela inclusiva: Concepciones, prácticas, profesorado y tecnologías". Nuances: estudos sobre Educação, Presidente Prudente-SP 26.2 (2015):

93-111. http://dx.doi.org/10.14572/nuances.v26i2.3398

- $\quad$ Rizales-Semprum, Mervin, Gómez-Valderrama, Carlos. and Hernández-Suarez, César. "Uso de herramientas tecnológicas para la enseñanza de las ciencias en educación media diversificada de acuerdo a la modalidad de estudio a distancia”. Eco matemático 10.2 (2019): 35-46. https://revistas.ufps.edu.co/index.php/ecomatematico/article/view/2591/2682

- Vargas-Murillo, Gavino. "Estrategias educativas y tecnología digital en el proceso enseñanza aprendizaje". Cuadernos 61.1 (2020): 69-76.

http://www.scielo.org.bo/pdf/chc/v61n1/v61n1_a10.pdf

RECIBIDO: 20/04/2021

APROBADO: 25/06/2021 